\title{
Requisitos Funcionais para Ambientes de Apoio ao Nível G do MPS.BR
}

\author{
Camilo Camilo Almendra ${ }^{1}$, Carla Ilane Moreira Bezerra ${ }^{1,2}$, Letícia Mara \\ Fernandes Nunes ${ }^{1}$, Emanuel Ferreira Coutinho ${ }^{2,3}$ \\ ${ }^{1}$ Campus de Quixadá - Universidade Federal do Ceará (UFC) \\ Av. José de Freitas Queiroz, 5.003 - Cedro - CEP 63900-000 - Quixadá - CE - Brasil \\ ${ }^{2}$ MDCC - Mestrado e Doutorado em Ciência da Computação \\ Universidade Federal do Ceará (UFC) - Fortaleza, CE - Brasil \\ ${ }^{3}$ Instituto UFC Virtual, Universidade Federal do Ceará (UFC), Fortaleza, CE - Brasil \\ \{camilo.almendra, carlailane\}@ufc.br, leticiamara.fernandes@gmail.com, \\ emanueldvirtual.ufc.br
}

\begin{abstract}
Software organizations have been adopting quality improvement models such as the Brazilian MPS.BR in order to enhance their product and service development processes. The adoption of process supporting tools is a critical success factor in software process improvement initiatives. This work aims at defining a set of functional requirements for supporting tools so that they can support processes adherent to MPS.BR level G. This set of requirements can aid the evaluation and selection of such tools. An evaluation of the Redmine tool is presented based of the reference requirements.
\end{abstract}

Resumo. Organizações desenvolvedoras de software estão adotando modelos de qualidade como o MPS.BR para a melhoria de seus processos, $e$ consequentemente seus produtos e serviços. A adoção de ambientes de apoio computacionais é um fator crítico de sucesso para iniciativas de melhoria de processos. Este trabalho tem como objetivo definir um conjunto de requisitos funcionais de referência para ambientes de apoio, que forneçam suporte a processos aderentes ao nível $G$ do MPS.BR. Esse conjunto de requisitos pode apoiar a seleção de ambientes de suporte. Uma avaliação da ferramenta Redmine é apresentada com base nos requisitos de referência.

\section{Introdução}

Cada vez mais as organizações de desenvolvimento de software estão adotando modelos de qualidade como o MPS.BR (Melhoria de Processo do Software Brasileiro) [Softex 2012] e o CMMI (Capability Maturity Model Integration) [Chrissis et al. 2011] para a melhoria de seus processos, e consequentemente seus produtos e serviços. A busca por qualidade vem em conjunto com uma competição local e global cada vez mais acirrada, o que exige das empresas a busca por excelência com desempenho e produtividade.

A implantação de uma iniciativa de melhoria de processo de software (MPS) é normalmente considerada de alto custo por muitas organizações, pois são necessários recursos significativos durante certo período de tempo. Desta forma, torna-se necessário 
desenvolver estratégias efetivas para a implantação de processos de software com sucesso [Montoni et al. 2008].

Para o sucesso de implantação desses processos é necessária a adoção de ferramentas de apoio computacional apropriadas que atendam tanto requisitos funcionais do modelo, quanto requisitos não-funcionais para a qualidade necessária da execução do processo. Segundo Santos (2008) é comum a identificação da necessidade de um ambiente de apoio adequado como um importante fator de sucesso em relatos relacionadas à execução de iniciativas de MPS [Niazi et al. 2005; Santos et al. 2007]. De fato, grande parte das organizações com baixos níveis de maturidade não possuem um ambiente adequado para iniciar a iniciativa [Santos et al. 2007].

As características dos ambientes de apoio devem estar alinhadas com os processos e os níveis de maturidade almejados pela organização. Uma mesma ferramenta pode fornecer suporte ao planejamento e/ou execução de processos de um determinado nível de maturidade, mas não ser suficiente para níveis posteriores ou outras áreas. São vários os trabalhos que apresentam avaliações ou comparações de ferramentas em relação aos modelos de maturidade MPS.BR e CMMI [Yoshidome et al. 2010; Yoshidome et al. 2012; Almeida et al. 2010]. No entanto, os trabalhos deixam implícitos os requisitos de alto nível inerentes aos modelos, e apontam diretamente funcionalidades de usuário nas ferramentas avaliadas. Para organizações iniciando iniciativas de MPS, é difícil a reutilização desses relatos, caso estejam realizando uma avaliação de aderências de ferramentas levando em conta o modelo MPS.BR e não um processo já definido.

Ambientes de apoio fornecem o suporte computacional às atividades de planejamento, gestão e execução dos processos de desenvolvimento e manutenção de software, constituindo apoio fundamental para a aderência ao processo de software pelas equipes de projetos [Schots et al. 2011]. Em iniciativas de melhoria de processo de software, a adoção de apoio computacional é considerada fator impactante e associada à redução de custos e de riscos [Montoni e Rocha 2010; Santos 2008; Almeida et al. 2011].

Uma dificuldade para novas empresas e profissionais que estão participando de iniciativas de melhoria é identificar quais são os requisitos funcionais necessários para que um ambiente forneça o suporte necessário ao processo. Ao analisar apenas o conjunto de Resultados Esperados e Atributos de Processo do MPS.BR, e cruzar essa informações com lista de características e funcionalidades das ferramentas, não há como estabelecer um mapeamento direto. Implicitamente, ao analisar uma ferramenta se verifica se as funcionalidades de coleta, processamento e extração de dados dando suporte às atividades e evidências tipicamente encontradas nos processos baseados no MPS.BR.

Nesse contexto, este trabalho propõe um guia com um conjunto de requisitos funcionais para o nível G do MPS.BR, que pode ser usado para avaliações de aderência de ferramentas. $O$ trabalho realizado visa contribuir para as organizações com um guia para apoiar o processo de seleção, aquisição e personalização de ambientes de apoio, dentro do contexto de iniciativas de melhoria que usem o MPS.BR como referência. Esse conjunto de requisitos pode apoiar a seleção de ambientes de suporte.

O restante deste artigo está organizado da seguinte forma: na Seção 2 são discutidos trabalhos relacionados; na Seção 3 é apresentada a metodologia desse 
trabalho; na Seção 4 são definidos requisitos para o ambientes no nível G do MPS.BR; na Seção 5 é realizada uma avaliação do ambiente de software livre Redmine baseado nos requisitos definidos e, na Seção 6 são apresentadas as conclusões e direções para trabalhos futuros.

\section{Trabalhos Relacionados}

Alguns ambientes de apoio ferramental a implantação e execução dos processos do MPS.BR podem ser destacados: WebAPSEE [Lima Reis et al. 2007], desenvolvida pelo LABES-UFPA, como ferramenta de apoio a gestão de processos de software; e Estação Taba [Rocha et al. 2005], um Ambiente de Desenvolvimento de Software (ADS) desenvolvida pela COPPE/UFRJ.

Ferramentas para a execução têm sido elaboradas ou adaptadas, de forma integrada a esses ambientes ou de forma isolada, para a execução de processos específicos nas organizações [Yoshidome et al. 2010; Yoshidome et al. 2012; Almeida et al. 2010]. Tanto os ambientes quanto as ferramentas isoladas desenvolvidas têm se mostrado como um diferencial para as organizações implantarem e executarem os processos de forma mais ágil e eficiente.

Young et al. (2006) descrevem uma experiência da aplicação do CMMI na melhoria dos processos de uma empresa através da seleção e integração de diversas ferramentas de software. Diversos estudos na literatura descreveram como ferramentas de software podem ser utilizadas para facilitar a adoção do CMMI [Cottrell 2006; Meisenbacher 2005]. Benefícios para a melhoria de processos CMMI através do emprego de ferramentas são apontados em [Cottrell 2006]. Ferramentas adequadas destinam-se a automatizar tarefas manuais e propensas a erros, além de facilitar e reforçar o controle de gestão, e aumentar a eficiência do processo qualidade do produto. Em relação à seleção de ferramentas, a fim de envolver atividades do processo CMMI, os seguintes tipos de ferramentas precisam ser avaliadas: planejamento, gerenciamento de requisitos, modelagem visual, desenvolvimento de código, interface de usuário, teste, gerenciamento de configuração e garantia da qualidade.

Para iniciativas que adotam o MR-MPS como modelo de qualidade, o conjunto de Resultados Esperados e Atributos de Processo do modelo servem como fonte para a tomada de decisão na definição do apoio computacional associado aos processos organizacionais e de desenvolvimento de software.

Em Yoshidome et al. (2010) e Yoshidome et al. (2012) são apresentadas propostas de implementação através de apoio automatizado para os resultados esperados dos processos de Gerência de Projetos e Desenvolvimento de Requisitos, respectivamente. Ambos os trabalhos relacionam cada um dos resultados esperados com recursos das ferramentas que compõe o pacote, e indicam evidências típicas para os resultados esperados do MPS.BR.

Guiar a avaliação de ambientes pelas evidências típicas alinha-se com a proposta do MR-MPS em identificar e avaliar os resultados gerados dos processos, ao invés de características específicas do processo, visto que diferentes processos podem gerar resultados equivalentes. No entanto, dentro de um contexto de iniciativa de MPS, os relatos da literatura não propiciam elementos reusáveis para que outras organizações avaliem ambientes de apoio diferentes, visto que os relatos apontam diretamente para recursos de interface e funcionalidades dos ambientes avaliados. 
Em Mendes et al. (2010), é apontada a ausência de especificação de requisitos para as ferramentas disponíveis no mercado. O trabalho estabeleceu requisitos de avaliação baseados nos processos definidos e em dificuldades já reportadas por equipes de uma empresa. No entanto, não há o estabelecimento de uma relação entre os requisitos e os resultados esperados do MPS.BR.

Neste contexto, esse trabalho propõe um conjunto de requisitos funcionais, relacionados com resultados esperados do MPS.BR para o nível $\mathrm{G}$, de forma a apoiar a avaliação de qualquer ambiente de apoio a processos de software.

\section{Metodologia}

O guia de requisitos funcionais foi elaborado a partir dos Atributos de Processo e Resultados Esperados do MPS.BR, sendo esses considerados a base dos objetivos de alto nível que qualquer ambiente de apoio a processos deve atender. Desses objetivos de alto nível, foram derivados objetivos intermediários representados em forma de requisitos funcionais. O estabelecimento e elaboração de objetivos em vários níveis é essencial em um processo de engenharia de requisitos, e a abordagem baseada em objetivos (goal-based) pode ser considerada uma maneira eficaz de atingir requisitos completos, evitar requisitos irrelevantes e promover o entendimento dos requisitos por stakeholders [Van Lamsweerde 2001].

A partir de cada objetivo de alto nível oriundo do modelo MPS.BR, foram derivados requisitos funcionais dos ambientes com foco na coleta, cruzamento e recuperação de informações necessárias para as atividades de acompanhamento e avaliação de processos. As informações necessárias que nortearam esse trabalho são aquelas tipicamente esperadas em evidências diretas coletadas em avaliação de processos, identificadas na experiência dos autores adquirida em projetos de melhoria e avaliação de processos em empresas e na pesquisa bibliográfica sobre ambientes de apoio a processos.

A partir de cada Resultado Esperado e Atributos de Processo, foram identificadas as informações essenciais que deveriam ser armazenáveis e recuperáveis de um ambiente de apoio a fim de que se pudesse garantir o atendimento do modelo MPS.BR. Os requisitos funcionais foram então elaborados em termos de capacidade de coletar, cruzar ou recuperar essas informações.

A fim de realizar uma validação do guia de requisitos elaborados, foi realizada uma avaliação da ferramenta Redmine, um software livre para gestão de projetos.

\section{Requisitos Funcionais para Ambientes de Apoio}

O MPS.BR [Softex 2012] é o programa para Melhoria de Processo do Software Brasileiro e define um modelo de referência para melhoria e avaliação de processos de software de forma a atender as necessidades de negócio de empresas brasileiras. O nível G do Modelo MPS.BR tem como foco os processos de Gerência de Projetos e Gerência de Requisitos.

O processo de Gerência de Projetos é responsável por estabelecer e manter planos, definir atividades, recursos e responsabilidades do projeto e corrigir desvios no desempenho do projeto e possui 19 resultados esperados. Já o Processo de Gerência de Requisitos tem como objetivo gerenciar os requisitos do produto e dos componentes do 
produto do projeto, e identificar inconsistências entre os requisitos, os planos do projeto e os produtos de trabalho do projeto. Possui 5 resultados esperados [Softex 2012].

O conjunto de requisitos funcionais apresentado nesse trabalho visa facilitar a seleção e avaliação de soluções para apoio computacional às iniciativas de MPS. O conjunto apresenta requisitos funcionais derivados dos Resultados Esperados e Atributos de Processo do MPS.BR, identificado assim características ou comportamentos que devem estar presentes e implementados de alguma forma em ferramentas avaliadas. O guia apresenta requisitos funcionais em termos de coleta, cruzamento e recuperação de informações tipicamente necessárias para evidenciar o uso do processo, sem entrar em detalhes de formato e apresentação dessas informações em interfaces de usuário e artefatos de entrada e saída de dados.

Cada resultado esperado dos processos de Gerência de Projetos (GPR) e Gerência de Requisitos (GRE) foi considerado um objetivo de alto nível a ser atendido. Nesse contexto, foram elaborados requisitos necessários para que uma ferramenta ou ambiente de apoio possa atender aos resultados esperados dos processos do nível G do MPS.BR. A Tabela 1 apresenta o conjunto de requisitos identificados em relação aos resultados esperados para o processo de Gerência de Projetos, e a Tabela 2 apresenta o conjunto relacionado com o processo de Gerência de Requisitos. Os requisitos que são identificados como Geral, requisitos RF-GPR-Ata e RF-GPR-Ação são requisitos que podem ser atribuídos a vários resultados esperados e não possuem um único resultado esperado associado aos mesmos.

Tabela 1. Requisitos funcionais para os resultados esperados do Nível G do MPS.BR para o processo Gerência de Projetos.

\begin{tabular}{|l|l|l|}
\hline $\begin{array}{l}\text { Resultado } \\
\text { Esperado }\end{array}$ & Requisito & Descrição \\
\hline Geral & $\begin{array}{l}\text { RF-GPR Ata } \\
\text { RF-GPR Ação }\end{array}$ & $\begin{array}{l}\text { Ata: Registrar uma ata de reunião } \\
\text { Ação: Registrar e acompanhar ação }\end{array}$ \\
\hline GPR1 & RF-GPR1.1 & $\begin{array}{l}\text { Manter registros unicamente identificados chamados de pacotes de } \\
\text { trabalho que representam as principais atividades a serem feitas no } \\
\text { projeto. }\end{array}$ \\
\hline GPR2 & $\begin{array}{l}\text { RF-GPR2.1 } \\
\text { RF-GPR2.2 } \\
\text { trabalhos definidos em RF-GPR1.1. } \\
\text { Estimar tamanho de cada tarefa a ser realizada. }\end{array}$ \\
\hline GPR3 & $\begin{array}{l}\text { RF-GPR3.1 } \\
\text { RF-GPR3.2 }\end{array}$ & $\begin{array}{l}\text { Documentar e publicar informações sobre o modelo de ciclo de vida } \\
\text { adotado no projeto. } \\
\text { Associar fases do ciclo de vida com execução do processo. }\end{array}$ \\
\hline RPR4 & $\begin{array}{l}\text { Ranter tarefas a serem feitas no cronograma do projeto. } \\
\text { RF-GPR4.2 } \\
\text { Consuciar a tarefas estimativas de tempo e estimativas de custo. }\end{array}$ \\
\hline GPR6 & RF-GPR5.2 & $\begin{array}{l}\text { Consultar custo total do projeto com base nas estimativas das tarefas. } \\
\text { Manter o cronograma do projeto. }\end{array}$ \\
\hline
\end{tabular}




\begin{tabular}{|c|c|c|}
\hline $\begin{array}{l}\text { Resultado } \\
\text { Esperado }\end{array}$ & Requisito & Descrição \\
\hline GPR7 & $\begin{array}{l}\text { RF-GPR7.1 } \\
\text { RF-GPR7.2 } \\
\text { RF-GPR7.3 }\end{array}$ & $\begin{array}{l}\text { Manter recursos humanos no projeto. } \\
\text { Vincular competências ou perfil a um recurso humano. } \\
\text { Consultar relatório com as competências da equipe. }\end{array}$ \\
\hline GPR8 & RF-GPR8.1 & Manter registros de recursos de infraestrutura. \\
\hline GPR9 & RF-GPR9.1 & $\begin{array}{l}\text { Configurar controle de acesso ao ambiente virtual dos projetos, } \\
\text { associando usuários e perfis a funções de criação ou consulta de dados. }\end{array}$ \\
\hline GPR10 & RF-GPR10.1 & $\begin{array}{l}\text { Gerar relatório integrado das informações planejadas em comparação } \\
\text { com o executado. }\end{array}$ \\
\hline GPR11 & RF-GRP11.1 & $\begin{array}{l}\text { Registro de estudos de viabilidade ou de revisões viabilizados através } \\
\text { do requisito RF-GPR-ATA. }\end{array}$ \\
\hline GPR12 & $\begin{array}{l}\text { RF-GPR12.1 } \\
\text { RF-GPR12.2 }\end{array}$ & $\begin{array}{l}\text { Divulgação do plano viabilizada através do RF-GPR10.1. } \\
\text { Obtenção de compromisso viabilizada através do RF-GPR-ATA. }\end{array}$ \\
\hline GPR13 & RF-GPR13.1 & $\begin{array}{l}\text { Comparar o escopo, tempo e custo estimado inicialmente com o } \\
\text { realizado. }\end{array}$ \\
\hline GPR14 & $\begin{array}{l}\text { RF-GPR14.1 } \\
\text { RF-GPR14.2 }\end{array}$ & $\begin{array}{l}\text { Comparar os recursos estimados inicialmente com o realizado. } \\
\text { Registro de desvios e observações viabilizado através dos requisitos } \\
\text { RF-GPR-ATA e RF-GPR-AÇÃO }\end{array}$ \\
\hline GPR15 & $\begin{array}{l}\text { RF-GPR15.1 } \\
\text { RF-GPR15.2 }\end{array}$ & $\begin{array}{l}\text { Monitoramento de riscos. } \\
\text { Acompanhamento de ações viabilizado através do RF-GPR-AÇÃO. }\end{array}$ \\
\hline GPR16 & RF-GPR16.1 & $\begin{array}{l}\text { Envolvimento das partes interessadas viabilizado através do RF-GPR- } \\
\text { ATA }\end{array}$ \\
\hline GPR17 & RF-GPR17.1 & Revisões dos marcos viabilizadas através do RF-GPR-ATA \\
\hline GPR18 & RF-GPR18.1 & Acompanhamento de problemas através do RF-GPR-AÇÃO. \\
\hline GPR19 & RF-GPR19.1 & $\begin{array}{l}\text { Acompanhamento de ações corretivas viabilizado através do RF-GPR- } \\
\text { AÇÃO }\end{array}$ \\
\hline
\end{tabular}

Tabela 2. Requisitos funcionais para os resultados esperados do Nível G do MPS.BR para o processo Gerência de Requisitos.

\begin{tabular}{|l|l|l|}
\hline $\begin{array}{l}\text { Resultado } \\
\text { Esperado }\end{array}$ & Requisito & Descrição \\
\hline Geral & $\begin{array}{l}\text { RF-GPR } \\
\text { RF-GPR }\end{array}$ & $\begin{array}{l}\text { Ata: Registrar uma ata de reunião } \\
\text { Ação: Registrar e acompanhar ação } \\
\text { Ganter requisitos e seu ciclo de vida. } \\
\text { Obtenção de comprometimento viabilizado através do RF-GPR-ATA. }\end{array}$ \\
\hline GRE2 & RF-GRE1.1 & $\begin{array}{l}\text { Apoiar revisão e avaliação dos requisitos. } \\
\text { Obtenção de comprometimento viabilizado através do RF-GPR-ATA. }\end{array}$ \\
\hline GRE3 & RF-GRE3.1 & $\begin{array}{l}\text { Associar requisito a produtos de trabalho impactados. } \\
\text { Associar requisito a outros requisitos impactados. }\end{array}$ \\
\hline GRE4 & RF-GRE4.1 & $\begin{array}{l}\text { Verificação de aderência dos requisitos com produtos de trabalho } \\
\text { viabilizada através dos RF-GRE2.1 e RF-GRE3.1. } \\
\text { Acompanhamento de ações viabilizado através do RF-GPR-AÇÃO. }\end{array}$ \\
\hline GRE5 & RF-GRE5.1 & Manter solicitações de mudança e seu ciclo de vida. \\
\hline
\end{tabular}

O verbo de ação "Manter" foi utilizado para cenários de CRUD (Create, Retrieve, Update, Delete). O requisito GPR10.1 é de natureza transversal, e complementa a avaliação de outras funcionalidades do sistema. A fim de fornecer suporte para uma gestão integrada, objetivo do resultado esperado GPR10, é necessário que as funcionalidades que viabilizam os demais requisitos possuam capacidade de manter um histórico ou baselines dos dados manipulados.

A capacidade de um processo é expressa pelo atendimento aos resultados esperados dos atributos de processo do nível G, RAP1 a RAP10. Alguns desses 
resultados podem ser monitorados e avaliados direta ou indiretamente através das evidências geradas pela automação dentro do ambiente de apoio dos demais requisitos funcionais, tais como RAP1, RAP5, RAP6 e RAP8. O atendimento do RAP2 envolve recursos de publicação e divulgação, que podem usar a infraestrutura comum a solução usada para atender os requisitos RF-GPR3.1. O RAP3 por sua vez pode ser viabilizado pelas soluções encontradas para os requisitos derivados dos resultados GPR3, GPR4, GPR5, GPR7 e GPR10. O RAP4 pode ser viabilizado por solução equivalente a RFGRE5. O RAP7 pode ser viabilizado pelas soluções usadas para os requisitos derivados do GPR7. Por fim, os RAP9 e RAP10 podem ser avaliadas através do conjunto de relatórios disponíveis e evidências coletadas durante a execução do processo apoiado pelo ambiente computacional. Com base nesses resultados esperados para os atributos de processos, foram identificados requisitos funcionais para os mesmos. Na Tabela 3 são listados os atributos de processos no nível G do MPS.BR e seus respectivos requisitos definidos.

Tabela 3. Requisitos para os Resultados Esperados dos Atributos de Processo no nível G do MPS.BR

\begin{tabular}{|l|l|}
\hline $\begin{array}{l}\text { Resultado } \\
\text { Esperado }\end{array}$ & Requisito \\
\hline RAP1 & Atingir os resultados do processo. \\
\hline RAP2 & Publicar políticas organizacionais. \\
\hline RAP3 & $\begin{array}{l}\text { Planejamento do processo viabilizado através dos requisitos RF-GPR3.1, RF-GPR3.2, } \\
\text { RF-GPR4.1, RF-GPR5.2, RF-GPR7.1, RF-GPR8.1, RF-GPR10.1. }\end{array}$ \\
\hline RAP4 & Monitorar a execução do processo com possíveis ajustes no mesmo. \\
\hline RAP5 & Identificar e disponibilizar as informações e os recursos do processo. \\
\hline RAP6 & Definir, atribuir e comunicar as responsabilidades do processo. \\
\hline RAP7 & Viabilizado pelos requisitos derivados do GPR7. \\
\hline RAP8 & Planejar e executar a comunicação entre as partes interessadas no processo. \\
\hline RAP9 & $\begin{array}{l}\text { Gerar relatório integrado sobre a execução do processo nos projetos. Depende das } \\
\text { diretrizes das políticas organizacionais. } \\
\text { Ações corretivas viabilizadas através do RF-AÇÃO. }\end{array}$ \\
\hline RAP10 & Viabilizado pelos Requisitos Gerais de Gerência de Projeto. \\
\hline
\end{tabular}

A elaboração do conjunto de requisitos ocorreu através da análise de evidências diretas típicas e as ações necessárias para que os resultados esperados pudessem ser observados em projetos de software. $\mathrm{O}$ conjunto de requisitos apresentado não representa a única forma de viabilizar os resultados esperados em uma iniciativa de MPS, já que é possível basear-se também em evidências indiretas para avaliar a maturidade de processos de uma organização.

Dentro de uma iniciativa de MPS de uma organização, na fase de seleção de ambientes de apoio, o conjunto de requisitos apresentado serve de guia para uma comparação funcional entre soluções. Ao apresentar uma listagem de requisitos funcionais essenciais, o processo de avaliação das soluções torna-se mais eficaz. Para que um ambiente atenda aos resultados esperados do Nível G, o mesmo deve fornecer soluções que atendam aos requisitos definidos.

Neste trabalho, não foram considerados objetivos relacionados ao apoio no planejamento e configuração de processos, presentes em ambientes como Estação TABA [Rocha et al. 2005] e WebAPSEE [Lima Reis e Reis 2006]. 


\section{Avaliação do Ambiente Redmine}

Nessa seção é apresentada uma avaliação de aderência da ferramenta Redmine, usando o conjunto de requisito funcionais da seção anterior como guia. A metodologia de avaliação consistiu dos seguintes passos:

- Análise da documentação do Redmine;

- Implantação de uma instância do produto em um ambiente de testes;

- Avaliação de aderência das características do produto aos requisitos levantados, através de consulta à documentação e simulação de uso na instância de testes.

$\mathrm{O}$ atendimento do requisito à ferramenta foi caracterizado seguindo os seguintes critérios: para os requisitos que foram atendidos complemente pela ferramenta foram classificados como Totalmente Implementado $(\mathrm{T})$, para os que não atendem ao requisito por completo foram classificados como Largamente Implementado (L), para os que atendem apenas a uma pequena parte do requisito foram classificados como Parcialmente Implementado $(\mathrm{P})$ e para os que não atendem ao requisito foram classificados como Não Implementado (N). Na coluna de justificativa é detalhado o motivo do requisito estar implementando o grau definido e o plugin correspondente que serviu para implementar o resultado esperado. Caso o requisito não implemente nenhum dos graus definidos, nenhuma explicação é realizada.

O objetivo do trabalho é demonstrar como o conjunto de requisitos pode apoiar a avaliação do grau de aderência de uma ferramenta ao nível $\mathrm{G}$, expondo as necessidades ausentes que podem ser viabilizadas através da extensão ou integração com outras soluções. A Tabela 4 apresenta o resultado da avaliação de aderência, com os respectivos graus de implementação e justificativa.

Tabela 4. Análise de aderência aos requisitos funcionais pela ferramenta Redmine.

\begin{tabular}{|c|c|c|}
\hline Requisito & Grau & Justificativa \\
\hline RF-GPR-Ata & $\mathrm{T}$ & $\begin{array}{l}\text { A adição da ata de reunião pode ser realizada no Redmine através da aba de } \\
\text { Documentos. Também é possível adicionar anexos aos documentos. } \\
\text { Plugin: não }\end{array}$ \\
\hline RF-GPR-Ação & $\mathrm{T}$ & $\begin{array}{l}\text { No cadastro de uma tarefa pode ser cadastrado um tipo de tarefa como } \\
\text { ação. } \\
\text { Plugin: não }\end{array}$ \\
\hline RF-GPR1.1 & $\mathrm{T}$ & $\begin{array}{l}\text { Na aba de Tarefas do Redmine é possível adicionar tarefas que representam } \\
\text { as } \\
\text { Plugin: não atividades a serem feitas no projeto. }\end{array}$ \\
\hline RF-GPR2.1 & $\mathrm{T}$ & $\begin{array}{l}\text { A partir de cada tarefa criada no Redmine é possível adicionar sub-tarefas } \\
\text { para a tarefa já criada. } \\
\text { Plugin: não }\end{array}$ \\
\hline RF-GPR2.2 & $\mathrm{N}$ & - \\
\hline RF-GPR3.1 & $\mathrm{T}$ & $\begin{array}{l}\text { Através da aba Wiki é possível documentar e disponibilizar um documento. } \\
\text { Também é possível fazer comentários sobre o documento. } \\
\text { Plugin: não }\end{array}$ \\
\hline RF-GPR3.2 & $\mathrm{T}$ & $\begin{array}{l}\text { O plugin Milestone adiciona marcos às tarefas do projeto. Os marcos podem } \\
\text { ser configurados como fase do ciclo de vida do projeto. } \\
\text { Plugin: http://www.redmine.org/plugins/redmine milestones }\end{array}$ \\
\hline RF-GPR4.1 & $\mathrm{L}$ & $\begin{array}{l}\text { Para cada tarefa, pode ser associado uma data de início e fim, e porcentagem } \\
\text { já realizada da tarefa. A partir do plugin MS Project é possível importar um } \\
\text { cronograma do Project. Porém um ponto fraco no plugin é que o mesmo não } \\
\text { é gratuito. Plugin: http://www.redmine.org/plugins/ms-project-import }\end{array}$ \\
\hline
\end{tabular}




\begin{tabular}{|c|c|c|}
\hline Requisito & Grau & Justificativa \\
\hline RF-GPR4.2 & $\mathrm{L}$ & $\begin{array}{l}\text { Para cada tarefa é possível adicionar uma estimativa de tempo em horas para } \\
\text { as mesmas. Com o Plugin Finance é possível criar custos para as operações } \\
\text { gastas no projeto. O plugin também faz a soma destas operações. Já o plugin } \\
\text { Budget-Sheet fornece a funcionalidade de associar gastos com as tarefas do } \\
\text { projeto. Porém, um ponto fraco no plugin é o que o mesmo não é gratuito. } \\
\text { Plugin: http://www.redmine.org/plugins/finance } \\
\text { http://www.redmine.org/plugins/payroll-budget-sheet }\end{array}$ \\
\hline RF-GPR4.3 & $\mathrm{L}$ & $\begin{array}{l}\text { Através do plugin Budget-Sheet é possível consultar os gastos com base nas } \\
\text { estimativas das tarefas. Os gastos dos outros projetos também são exibidos. } \\
\text { Porém, um ponto fraco no plugin é o que o mesmo não é gratuito. } \\
\text { Plugin: http://www.redmine.org/plugins/payroll-budget-sheet }\end{array}$ \\
\hline RF-GPR5.1 & $\mathrm{L}$ & $\begin{array}{l}\text { O custo das tarefas pode ser verificado através do plugin Budget-Sheet. } \\
\text { Porém, um ponto fraco no plugin é o que o mesmo não é gratuito. } \\
\text { Plugin: http://www.redmine.org/plugins/payroll-budget-sheet }\end{array}$ \\
\hline RF-GPR5.2 & $\mathrm{P}$ & $\begin{array}{l}\text { É possível verificar o andamento do cronograma do projeto através do } \\
\text { gráfico de Gantt ou através do plugin MS Project. Porém não é possível } \\
\text { fazer a relação dos custos junto com as tarefas no cronograma. } \\
\text { Plugin: http://www.redmine.org/plugins/ms-project-import }\end{array}$ \\
\hline RF-GPR6.1 & $P$ & $\begin{array}{l}\text { Através da customização de tipos de tarefas e campos das tarefas é possível } \\
\text { trata-la como risco, porém não é fornecido alguns campos que calculem a } \\
\text { prioridade do risco com base na probabilidade e impacto deste. } \\
\text { Plugin: não }\end{array}$ \\
\hline RF-GPR7.1 & $\underline{\mathrm{P}}$ & $\begin{array}{l}\text { É possível adicionar usuários e associa-los a tarefas do projeto. É possível } \\
\text { também associar papeis e definir grupos para os usuários. Porém não é } \\
\text { possível fazer o planejamento desses usuários. } \\
\text { Plugin: não }\end{array}$ \\
\hline RF-GPR7.2 & $\underline{P}$ & $\begin{array}{l}\text { Com o plugin People é possível inserir informações complementares para } \\
\text { cada usuário. Porém, essas informações não evidenciam as competências ou } \\
\text { perfil de cada recurso humano. } \\
\text { Plugin: http://www.redmine.org/plugins/people }\end{array}$ \\
\hline RF-GPR7.3 & $\underline{\mathrm{N}}$ & tres \\
\hline RF-GPR8.1 & $\mathrm{P}$ & $\begin{array}{l}\text { É possível adicionar registros de alguns equipamentos através do plugin } \\
\text { Equipment. Porém os recursos adicionados não podem ser alocados no } \\
\text { projeto. } \\
\text { Plugin: http://www.redmine.org/plugins/redmine equipment status viewer }\end{array}$ \\
\hline RF-GPR9.1 & $\underline{T}$ & $\begin{array}{l}\text { É possível adicionar e configurar permissões de papéis e responsabilidades } \\
\text { no projeto para o acesso as informações. } \\
\text { Plugin: não }\end{array}$ \\
\hline RF-GPR10.1 & $\mathrm{N}$ & - \\
\hline RF-GPR11.1 & $\mathrm{L}$ & $\begin{array}{l}\text { Através das atas de reuniões é possível identificar a viabilidade do projeto. } \\
\text { Porém, não é definido uma funcionalidade específica para verificar a } \\
\text { viabilidade. } \\
\text { Plugin: não }\end{array}$ \\
\hline RF-GPR12.1 & $\underline{T}$ & $\begin{array}{l}\text { É possível divulgar qualquer documento através da aba de Documentos ou } \\
\text { pela Wiki. } \\
\text { Plugin: não }\end{array}$ \\
\hline RF-GPR12.2 & $\mathrm{T}$ & $\begin{array}{l}\text { É possível acompanhar o compromisso dos membros do projeto através de } \\
\text { Atas de Reunião. } \\
\text { Plugin: não }\end{array}$ \\
\hline RF-GPR13.1 & $\underline{\mathrm{N}}$ & - \\
\hline RF-GPR14.1 & $\mathrm{N}$ & - \\
\hline RF-GPR14.2 & $\mathrm{T}$ & $\begin{array}{l}\text { É possível registrar desvios e observações no projeto através de atas ou } \\
\text { ações do projeto. } \\
\text { Plugin: não }\end{array}$ \\
\hline
\end{tabular}




\begin{tabular}{|c|c|c|}
\hline Requisito & Grau & Justificativa \\
\hline RF-GPR15.1 & $\mathrm{N}$ & - \\
\hline RF-GPR15.2 & $\mathrm{P}$ & $\begin{array}{l}\text { As ações podem ser acompanhadas através do cadastro de uma tarefa do } \\
\text { tipo Ação. Porém as mesmas não estarão associadas a riscos do projeto. } \\
\text { Plugin: não }\end{array}$ \\
\hline RF-GPR16.1 & $\mathrm{T}$ & $\begin{array}{l}\text { É possível acompanhar o envolvimento dos interessados no projeto através } \\
\text { das atas de reuniões. O planejamento e a comunicação com as partes } \\
\text { interessadas pode ser realizado por meio da Ata de reunião. } \\
\text { Plugin: não }\end{array}$ \\
\hline RF-GPR17.1 & $\mathrm{T}$ & $\begin{array}{l}\text { As revisões nos marcos do projeto podem ser documentadas através de atas } \\
\text { de reuniões. } \\
\text { Plugin: não }\end{array}$ \\
\hline RF-GPR18.1 & $\mathrm{T}$ & $\begin{array}{l}\text { Os problemas do projeto podem ser acompanhados através de tarefas com o } \\
\text { tipo cadastrado problema. } \\
\text { Plugin: não }\end{array}$ \\
\hline RF-GPR19.1 & $\mathrm{L}$ & $\begin{array}{l}\text { As ações corretivas para os problemas podem ser adicionadas através de } \\
\text { tarefas do tipo cadastrado ação. Porém não é possível fazer a ligação de uma } \\
\text { ou mais ações aos problemas. } \\
\text { Plugin: não }\end{array}$ \\
\hline RF-GRE1.1 & $P$ & $\begin{array}{l}\text { No Redmine os requisitos podem ser adicionados através de uma nova tarefa } \\
\text { com a categoria funcionalidade. Porém ele não fornece uma forma de } \\
\text { verificar se o requisito está validado pelo cliente. }\end{array}$ \\
\hline RF-GRE2.1 & $\mathrm{L}$ & $\begin{array}{l}\text { No Redmine através do plugin Checklist é possível adicionar critérios para } \\
\text { cada tarefa do projeto, mas o plugin não fornece uma opção de adicionar os } \\
\text { mesmos checklists para todas as tarefas. } \\
\text { Plugin: http://www.redmine.org/plugins/issue checklist }\end{array}$ \\
\hline $\begin{array}{l}\text { RF-GRE3.1 } \\
\text { RF-GRE3.2 }\end{array}$ & $\mathrm{N}$ & - \\
\hline $\begin{array}{l}\text { RF-GRE4.1 } \\
\text { RF-GRE4.2 }\end{array}$ & $\mathrm{P}$ & $\begin{array}{l}\text { O plugin Checklist permite adicionar critérios para cada tarefa do projeto. } \\
\text { Porém não foi encontrada nenhuma funcionalidade que permita fazer uma } \\
\text { revisão nos requisitos e incluir inconsistências. } \\
\text { Plugin: http://www.redmine.org/plugins/issue checklist }\end{array}$ \\
\hline RF-GRE5.1 & $\mathrm{N}$ & - \\
\hline
\end{tabular}

A avaliação feita na ferramenta é importante para identificar as possíveis necessidades da ferramenta com o processo de Gerência de Projetos no nível G do MPS.BR. A partir das necessidades levantadas com a ferramenta é possível perceber que esta necessita de algumas funcionalidades não encontradas na ferramenta para que esta atenda aos requisitos definidos para a implementação do processo de Gerência de Projetos no nível G do MPS.BR.

A avaliação dos atributos de processo não foi realizada no Redmine, pois os mesmos estão cobertos pelos requisitos dos resultados esperados do processo de Gerência de Projetos. Após a avaliação dos requisitos definidos, foi feita a contagem dos resultados obtidos. Com base na avaliação dos requisitos definidos junto com a implementação dos mesmos na ferramenta Redmine foram selecionados os requisitos correspondentes a risco do projeto para serem desenvolvidos em forma de plugin para a ferramenta.

\section{Conclusão e Trabalhos Futuros}

Esse trabalho apresentou um conjunto de requisitos funcionais voltados para apoiar o processo de seleção de ambientes de apoio para iniciativas de melhoria de processo de 
software baseadas no MPS.BR. Tais iniciativas possuem a adoção de apoio computacional como fator importante de sucesso, sendo a seleção de ambientes considerada uma etapa essencial em modelos de melhoria de processo de software.

Esse trabalho baseou-se em evidências diretas típicas para modelar um conjunto de requisitos funcionais aderente aos resultados esperados do MPS.BR. Foi adotada uma abordagem de derivação de requisitos funcionais baseados em objetivos de alto nível. Não foram apontados papéis específicos nos requisitos, visto que a responsabilidade pela execução dos processos varia de acordo com cada organização, e o modelo MPS.BR não prevê papéis específicos.

A fim de demonstrar o uso do conjunto de requisitos na avaliação de um ambiente, foi apresentada a avaliação da ferramenta Redmine. A avaliação do Redmine teve como objetivo apresentar uma análise do atendimento das funcionalidades da ferramenta com os resultados dos processos do nível G do MPS.BR.

Esse conjunto de requisitos pode ser utilizado por organizações em processo de seleção de ambientes de apoio para processos de software, assim com para guiar iniciativas de criação ou evolução de ambientes já existentes. Como trabalhos futuros, destacam-se abordar outros aspectos de qualidade de produto tais como usabilidade, eficiência e manutenibilidade, assim como a expansão da definição dos requisitos para os resultados esperados dos processos do nível F do MPS.BR.

\section{Referências}

Almeida, G., Ramos, B., Neto, M., Reis, M., Barcelos, M., Vasconcelos, A. (2010) "Ferramenta de Apoio à Engenharia de Requisitos Integrada a um Ambiente Colaborativo de Código Aberto". In: Congresso Brasileiro de Software: Teoria e Prática (CBSoft), Sessão de Ferramentas - Vol. 4, Salvador, BA, Brasil.

Almeida, C. D. A., Macedo, T. C., Albuquerque, A. B. (2011) "A continuidade da execução dos processos de software em empresas avaliadas no MPS.BR". In: X Simpósio Brasileiro de Qualidade de Software (SBQS), pp. 135-149, Curitiba, Brasil.

Chrissis, M., Konrad, M., Shrum, S. (2011) "CMMI Guidelines for Process Integration and Product Improvement". Addisson-Wesley, EUA, 3rd Edition.

Cottrell, B. (2006) "Tools and Process Maturity", http://www.saspin.org/Saspin_May2002_Cottrell.pdf, accessed 2006/11/8.

Lima, A., Costa, A., França, B., Reis, C. A., Reis, R. Q. (2006). "Gerência Flexível de Processos de Software com o Ambiente WebAPSEE". 20 Simpósio Brasileiro de Engenharia de Software-Sessão de Ferramentas. Florianópolis, Brasil.

Meisenbacher, L. K. (2005) "The Challenges of Tool Integration for Requirements Engineering", Proceedings of SREP'05, 2005, pp. 188-191.

Mendes, F. F., Fernandes, P. G., de Oliveira, J. L., da Cunha Mota, C., Martins, M. D. S., da Silva, R. (2010). "Análise de Ferramentas para Apoio à Gerência de Projetos e Gerência de Requisitos de Software". In: VI Workshop Anual do MPS, WAMPS 2010, pp. 148-157, Campinas, Brasil. 
Montoni, M.; Santos, G.; Katsurayama, A.; Cabral, R.; Figueiredo, S.; Natali, A. (2008) "Aplicação da Estratégia SPI-KM para Apoiar a Implementação do MPS.BR Níveis G e F em Pequenas e Médias Empresas do Rio de Janeiro", In: $7^{\circ}$ Simpósio Brasileiro de Qualidade de Software (SBQS'08). Florianópolis, SC, Brasil.

Montoni, M.; Rocha, A. R. (2010) "Aplicação de Grounded Theory para Investigar Iniciativas de Implementação de Melhorias em Processos de Software". In: $I X$ Simpósio Brasileiro Qualidade de Software (SBQS 2010), pp. 167-181, Belém, PA.

Niazi, M., Wilson, D., Zowghi, D., 2005, "A framework for assisting the design of effective software process improvement implementation strategies", In: Journal of Systems and Software, v. 78, n. 2, pp. 204-222.

Rocha, A. R., Montoni, M., Santos, G., Mafra, S., Figueiredo, S., Bessa, A. (2005) "Estação TABA: Uma Infraestrutura para Implantação do Modelo de Referência para Melhoria de Processo de Software", In: Simpósio Brasileiro de Qualidade de Software, pp. 49-60, Brasília, Brasil.

Santos, G., Montoni, M., Figueiredo, S., Rocha, A. R. (2007). "SPI-KM-lessons learned from applying a software process improvement strategy supported by knowledge management". In: Product-Focused Software Process Improvement, pp. 81-95. Springer Berlin Heidelberg.

Santos, G. (2008) "Ambientes de Engenharia de Software Orientados a Corporação". Tese de Doutorado, Rio de Janeiro, COPPE/UFRJ.

Schots, N. C. L., Santos, G., Cerdeiral, C., Cabral, M., Cabral, R., Schots, M., Nunes, E.,Rocha, A. R. (2011) "Lições Aprendidas em Implementações de Melhoria de Processos em Organizações com Diferentes Características”. In: VII Workshop Anual do MPS, WAMPS 2011, pp. 84-93, Campinas, Brasil.

Softex (2012) "MPS.BR: Melhoria de Processo do Software Brasileiro - Guia Geral", Disponível em: http://www.softex.br/mpsbr.

Van Lamsweerde, A. (2001) "Goal-oriented requirements engineering: a guided tour". In: Proceedings of Fifth IEEE International Symposium on Requirements Engineering. pp. 249-262, Toronto, Canadá.

Yoshidome, E.Y.C., Souza, M. R. A., Lira, W. M. P., Oliveira, S. R. B. (2010) "Uma Implementação do Processo de Gerência de Projetos Usando Ferramentas de Software Livre". In: Anais do VI Workshop Anual do MPS.BR - WAMPS, pp. 212219, Campinas, Brasil.

Yoshidome, E. Y. C., Ronny, M., Lira, W., Oliveira, S. R. B., Vasconcelos, A. M. L. (2012) "Uma Proposta de Apoio Sistematizado à Implementação do Processo de Desenvolvimento de Requisitos do MPS.BR e CMMI a partir do Uso de Ferramentas de Software Livre". In: Workshop on Requirements Engineering - WER, Buenos Aires, Argentina.

Young, H.; Fang, T.; Hu, C. (2006) "A Successful Practice of Applying Software Tools to CMMI Process Improvement" In: Journal of Software Engineering Studies, Vol. 1, No. 2, 78-95. 